\title{
Flavour violating squark and gluino decays
}

\author{
Tobias Hurth $^{a, b}$ and Werner Porod ${ }^{c}$ \\ ${ }^{a}$ CERN, Dept. of Physics, Theory Division, \\ CH-1211 Geneva, Switzerland \\ ${ }^{b} S L A C$, Stanford University, \\ Stanford, CA 94309, U.S.A. \\ ${ }^{c}$ Institut für Theoretische Physik und Astrophysik, Univ. Würzburg, \\ D-97074 Würzburg, Germany \\ E-mail: Tobias.Hurth@cern.ch, porod@physik.uni-wuerzburg.de
}

\begin{abstract}
We consider scenarios with large flavour violating entries in the squark mass matrices focusing on the mixing between second and third generation squarks. These entries govern both, flavour violating low energy observables on the one hand and squark and gluino decays on the other hand. We first discuss the constraints on the parameter space due to the recent data on $B$ mesons from the $B$ factories and Tevatron. We then consider flavour violating squark and gluino decays and show that they can still be typically of order $10 \%$ despite the stringent constraints from low energy data. Finally we briefly comment on the impact for searches and parameter determinations at future collider experiments such as the upcoming LHC or a future International Linear Collider.
\end{abstract}

KEYwORds: Supersymmetry Phenomenology

ArXiv EPrint: 0904.4574 


\section{Contents}

1 Introduction 1

2 Preliminaries $\quad 2$

2.1 Decays of squarks and gluinos 2

2.2 Flavour changing neutral currents 3

3 Phenomenological analysis $\quad 5$

3.1 Benchmark points, experimental and theoretical constraints 5

$\begin{array}{lll}3.2 & \text { Results } & 7\end{array}$

$\begin{array}{lll}3.2 .1 & \text { Constraints on flavour parameters } & 7\end{array}$

$\begin{array}{lll}\text { 3.2.2 Flavour violating decays of squarks and gluinos } & 8\end{array}$

4 Impact for LHC and ILC 13

5 Conclusions $\quad 15$

\section{Introduction}

Theoretical arguments like the hierarchy problem motivate the general expectation that the experiments at the LHC will lead to discoveries of new degrees of freedom at the TeV energy scale. The precise nature of this new physics is unknown, but it most probably will answer some of the fundamental questions related to the origin of electroweak symmetry breaking.

Rare $B$ and kaon decays (for a review see $[1,2]$ ) representing loop-induced processes are highly sensitive probes for new degrees of freedom beyond the SM establishing an alternative way to search for new physics. However, this indirect search for new physics signatures within flavour physics takes place today in complete darkness, given that we presently have no direct evidence of new particles beyond the Standard Model (SM). But the day the existence of new degrees of freedom is established by the Large Hadron Collider (LHC), the searches for anomalous phenomena in the flavour sector will become mandatory. The problem then will no longer be to discover new physics, but to measure its (flavour) properties.

Thus, within the next decade an important interplay of flavour and high- $p_{T}$ physics most probably will take place. For example, within supersymmetric extensions of the SM, the measurement of the flavour structure is directly linked to the crucial question of the supersymmetry-breaking mechanism as the soft SUSY breaking terms are the source of flavour structures beyond the SM. LHC has the potential to discover strongly interacting supersymmetric particles up to a scale of $2 \mathrm{TeV}$ and to measure several of their properties [3-6]. This information can be used for a refined analysis of flavour physics observables indicating possible flavour structures and, thus, give important information for distinguishing between models of supersymmetry breaking. 
Data from $K$ and $B_{d}$ physics show that new sources of flavour violation in $s \rightarrow d$ and $b \rightarrow d$ are strongly constrained, while the possibility of sizable new contributions to $b \rightarrow s$ remains open. We also have hints from model building: flavour models are not very effective in constraining the $b \rightarrow s$ sector [7]. Moreover, in SUSY-GUTs the large mixing angle in the neutrino sector relates to large mixing in the right-handed $b$-s sector [8-10].

As we explicitly show in this paper, such flavour information on the $b \rightarrow s$ observables is complementary to the high- $p_{T}$ data of the LHC. In fact, squark and gluino decays are governed by the same mixing matrices as the contributions to flavour violating loop transitions. This allows for possible direct correlations between flavour non-diagonal observables in $B$ and high- $p_{T}$ physics. We already anticipate that the present bounds on squark mixing, induced by the low-energy data on $b \rightarrow s$ transitions, still allow for large contributions to flavour violating squark decays at tree level. Due to the restrictions in flavour tagging at the LHC, additional information from future flavour experiments will be necessary to interpret those LHC data properly. Also the measurement of correlations between various squark decay modes at a future ILC would provide information about the flavour violating parameters.

Flavour violating squark and gluino decays have already been considered some time ago [11]. In the present paper we generalize and update the previous analysis including additional experimental constraints. More recently, similar work on the charged Higgs boson production was presented; it was shown that squark mixing can significantly change the production pattern [12]. In another work the interesting question was addressed if high- $p_{T}$ data can contribute to the solution of the flavour problem $[13,14]$. Recently, it was shown that one can also derive significant bounds on flavour-violating parameters of the squark sector by requiring that the radiative corrections to the CKM elements do not exceed the experimental values [15]. Flavour violating squark production has been studied in [16-18] where it has been found that flavour violating production can be sizable after taking into account constraints from the $b \rightarrow s$ observables and the anomalous magnetic moment of the muon.

The paper is organized as follows: in section 2 we review the necessary information about flavour mixing in the MSSM and about decays of squarks and gluinos in order to introduce our notation. In section 3 we present our phenomenological results and in section 4 we discuss their impact for LHC and ILC.

\section{Preliminaries}

\subsection{Decays of squarks and gluinos}

In the study of squark decays two limiting scenarios can be distinguished depending on the SUSY spectrum:

1. $m_{\tilde{g}}>m_{\tilde{q}_{i}}(q=d, u ; i=1, \ldots, 6)$ : in this case the gluino will mainly decay according to

$$
\tilde{g} \rightarrow d_{j} \tilde{d}_{i}, \quad \tilde{g} \rightarrow u_{j} \tilde{u}_{i}
$$


with $d_{j}=(d, s, b)$ and $u_{j}=(u, c, t)$ followed by squark decays into neutralino and charginos

$$
\tilde{u}_{i} \rightarrow u_{j} \tilde{\chi}_{k}^{0}, d_{j} \tilde{\chi}_{l}^{+}, \tilde{d}_{i} \rightarrow d_{j} \tilde{\chi}_{k}^{0}, u_{j} \tilde{\chi}_{l}^{-}
$$

In addition there can be decays into gauge and Higgs bosons if kinematically allowed:

$$
\tilde{u}_{i} \rightarrow Z \tilde{u}_{k}, H_{r}^{0} \tilde{u}_{k}, W^{+} \tilde{d}_{j}, H^{+} \tilde{d}_{j} ; \quad \tilde{d}_{i} \rightarrow Z \tilde{d}_{k}, H_{r}^{0} \tilde{d}_{k}, W^{-} \tilde{u}_{j}, H^{-} \tilde{u}_{j}
$$

where $H_{r}^{0}=\left(h^{0}, H^{0}, A^{0}\right), k<i, j=1, \ldots, 6$. Note, that due to the fact that there is left-right mixing in the sfermion mixing, one has flavour changing neutral decays into $Z$-bosons at tree-level.

2. $m_{\tilde{g}}<m_{\tilde{q}_{i}}(q=d, u ; i=1, \ldots, 6)$ : in this case the squarks decay mainly into a gluino,

$$
\tilde{u}_{i} \rightarrow u_{j} \tilde{g}, \quad \tilde{d}_{i} \rightarrow d_{j} \tilde{g}
$$

and the gluino decays via three-body decays and loop-induced two-body decays into charginos and neutralinos

$$
\tilde{g} \rightarrow d_{j} d_{i} \tilde{\chi}_{k}^{0}, u_{j} u_{i} \tilde{\chi}_{k}^{0}, \quad \tilde{g} \rightarrow u_{j} d_{i} \tilde{\chi}_{l}^{ \pm}, \quad \tilde{g} \rightarrow g \tilde{\chi}_{k}^{0}
$$

with $i, j=1,2,3, l=1,2$ and $k=1,2,3,4$. Note that the first two decay modes may contain states with quarks of different generations of quarks.

Of course, there is also the mixed case when the gluino mass lies within the squark mass spectrum. In such a case both, gluinos and squarks, will mainly decay into two-body final states. This is for example the case for benchmark point $\gamma$ sudied in section 3 .

Explicit formulas for the partial widths including flavour effects can be found in ref. [17]. The flavour mixing final states of the decays listed above are constrained by the fact that all observed phenomena in rare meson decays are consistent with the SM predictions. As we will show in the next sections, there are regions in the parameter space where the flavour violating decay modes can be even of the order of $10 \%$.

\subsection{Flavour changing neutral currents}

Within the Minimal Supersymmetric Standard Model (MSSM) there are two new sources of flavour changing neutral currents (FCNC), namely new contributions which are induced through the quark mixing as in the SM and generic supersymmetric contributions through the squark mixing. The latter is described by their mass matrices,

$$
\mathcal{M}_{f}^{2} \equiv\left(\begin{array}{cc}
M_{f, L L}^{2}+F_{f L L}+D_{f L L} & M_{f, L R}^{2}+F_{f L R} \\
\left(M_{f, L R}^{2}\right)^{\dagger}+F_{f R L}^{*} & M_{f, R R}^{2}+F_{f R R}+D_{f R R}
\end{array}\right)
$$

where $f$ stands for up- or down-type squarks. In the super-CKM basis in which the quark mass matrix is diagonal they read as

$$
D_{f L L}=\left(T_{3, f}-e_{f} \sin ^{2} \theta_{W}\right) \cos (2 \beta) m_{Z}^{2}, \quad D_{f R R}=e_{f} \sin ^{2} \theta_{W} \cos (2 \beta) m_{Z}^{2}
$$


for the $D$-terms,

$$
F_{f L L, i j}=F_{f R R, i j}=m_{i}^{2} \delta_{i j}, \quad F_{f R L, i j}=-\mu m_{i} \delta_{i j}(\tan \beta)^{-2 T_{3, f}}
$$

where $m_{i}$ are the corresponding quark masses, $e_{f}$ the electric charge, and $T_{3, f}$ the weak isospin of the corresponding left-handed quark. In this basis the $F$ - and $D$-terms are flavour diagonal and all flavour violation beyond the CKM resides in the soft SUSY breaking terms:

$$
\begin{array}{lll}
M_{d, L L}^{2}=V_{C K M}^{\dagger} M_{u, L L}^{2} V_{C K M}=\hat{m}_{\tilde{Q}}^{2} \equiv V_{d}^{\dagger} m_{\tilde{Q}}^{2} V_{d}, \\
M_{d, R R}^{2}=\hat{m}_{\tilde{d}}^{2} \equiv U_{d}^{\dagger} m_{\tilde{d}}^{2^{T}} U_{d}, & M_{u, R R}^{2}=\hat{m}_{\tilde{u}}^{2} \equiv U_{u}^{\dagger} m_{\tilde{u}}^{2 T} U_{u}, \\
M_{d, L R}^{2}=v_{1} / \sqrt{2} \hat{T}_{D} \equiv v_{1} / \sqrt{2} U_{d}^{\dagger} T_{D}^{T} V_{d}, & & M_{u, L R}^{2}=v_{2} / \sqrt{2} \hat{T}_{U} \equiv v_{2} / \sqrt{2} U_{u}^{\dagger} T_{U}^{T} V_{u},
\end{array}
$$

where the un-hatted mass matrices $m_{Q, u, d}^{2}$ and trilinear interaction matrices $T_{U, D}$ are given in the electroweak basis. The transformations $V_{u, d}$ and $U_{u, d}$ just bring the quarks from the interaction eigenstate basis to their mass eigenstate basis, so $V_{C K M}=V_{u}^{\dagger} V_{d}$. The relation between $M_{u, L L}$ and $M_{d, L L}$ is due to $\mathrm{SU}(2)_{L}$ gauge invariance. The $T$-matrices are in general non-hermitian. ${ }^{1}$

These additional flavour structures induce flavour violating couplings to the charginos, neutralinos and gluinos in the mass eigenbasis, which give rise to additional contributions to observables in the $K$ and $B$ meson sector. The low-energy observables can thus be used to constrain the size of the off-diagonal elements of the mass matrices $M_{f, L L}^{2}, M_{f, R R}^{2}$, and $M_{f, L R}^{2}$. Some of the flavour-violating terms may turn out to be poorly constrained. Thus, it is suitable to rely on the mass eigenstate formalism, which remains valid - in contrast to the mass insertion approximation - even when the intergenerational mixing elements are large. The diagonalization of the two $6 \times 6$ squark mass matrices squared $\mathcal{M}_{d}^{2}$ and $\mathcal{M}_{u}^{2}$ yields the eigenvalues $m_{\tilde{d}_{k}}^{2}$ and $m_{\tilde{u}_{k}}^{2}(k=1, \ldots, 6)$. The corresponding mixing matrices $R_{i j}^{f}$ relate the mass eigenstates with the electroweak eigenstates

$$
\tilde{u}_{k}=R_{k j}^{u} \tilde{u}_{j}^{e w}, \tilde{d}_{k}=R_{k j}^{d} \tilde{d}_{j}^{e w}
$$

where $\tilde{u}_{j}^{e w} \in\left\{\tilde{u}_{L}, \tilde{c}_{L}, \tilde{t}_{L}, \tilde{u}_{R}, \tilde{c}_{R}, \tilde{t}_{R}\right\}$ and $\tilde{d}_{j}^{e w} \in\left\{\tilde{d}_{L}, \tilde{s}_{L}, \tilde{b}_{L}, \tilde{d}_{R}, \tilde{s}_{R}, \tilde{b}_{R}\right\}$, respectively.

As usual, the flavour off-diagonal elements of the squark mass matrices are normalized by the diagonal ones. One uses the average of the diagonal elements (trace of the mass matrix divided by six) in the up and down sector, denoted by $m_{\tilde{\tilde{q}}}^{2}$. The observables can then be studied as a function of the normalized off-diagonal elements

$$
\begin{aligned}
\delta_{L L, i j}=\frac{\left(M_{f, L L}^{2}\right)_{i j}}{m_{\tilde{q}}^{2}}, & \delta_{f, R R, i j}=\frac{\left(M_{f, R R}^{2}\right)_{i j}}{m_{\tilde{f}}^{2}}, \quad(i \neq j) \\
\delta_{f, L R, i j}=\frac{\left(M_{f, L R}^{2}\right)_{i j}}{m_{\tilde{f}}^{2}}, & \delta_{f, R L, i j}=\frac{\left(M_{f, R L}^{2}\right)_{i j}^{\dagger}}{m_{\tilde{f}}^{2}} .
\end{aligned}
$$

where $f$ is either $u$ or $d$ for $u$-squarks and $d$-squarks, respectively. We emphasize that a consistent analysis of the bounds should also include interference effects between the various contributions, namely the interplay between the various sources of flavour violation and the interference effects of SM and various new-physics contributions. (see ref. [20]).

\footnotetext{
${ }^{1}$ We follow here the conventions of the SUSY Les Houches Accord, for more details see ref. [19].
} 
At present, new physics contributions to $s \rightarrow d$ and $b \rightarrow d$ transitions are strongly constrained. In particular, the transitions between first- and second-generation quarks, namely FCNC processes in the $K$ system, lead to very strong constraints on the parameter space of various new physics models. However, most of the phenomena involving $b \rightarrow s$ transitions are still largely unexplored and leave open the possibility of large new physics effects, in spite of the strong bounds of the three most important $b \rightarrow s$ observables, namely, the inclusive decay modes $\bar{B} \rightarrow X_{s} \gamma$ and $\bar{B} \rightarrow X_{s} \ell^{+} \ell^{-}$and the $B_{s}-\bar{B}_{s}$ mixing. It is well-known that the gluino contributions to these two inclusive decay modes are mostly sensitive to $\delta_{d, 23, L R}$ and $\delta_{d, 23, R L}$ in the leading order approximation [21-23]. However, the restriction to a single $\delta$ parameter and to the gluino contribution lead to unrealistically strong constraints as was already pointed out in ref. [20]. There are also sensitivity to combinations of other $\delta$ parameters and, moreover, depending on the precise choice of the supersymmetric flavour- diagonal parameters, other supersymmetric contributions which are also sensitive up-squark mixing parameters. The gluino contributions to $B_{s}-\bar{B}_{s}$ mixing are mainly sensitive to the combination $\delta_{L L} \delta_{d, R R}$ and $\delta_{d, L R} \delta_{d, R L}$. The latter is not really relevant due to the constraints already induced by the inclusive decay modes. But also here sensitivity of that observable to other squark mixing parameters are not negligible.

Moreover, there is an impact of squark mixing including the third generation on the lightest Higgs mass and the $\rho$ parameter which also includes the $\delta$ parameter in the upsquark sector (see for example [24]). There are the Tevatron bounds on squark masses and certain constraints from dark matter phenomenology. Also the diagonal $T$ entries (see eq. 2.11) have to be tuned that they lead to perturbatively quark masses. Finally, we recall that there are also constraints on some $\delta$ parameters from theoretical considerations, namely that one should avoid colour and charge breaking minima [25]. All resulting numerical bounds and constraints will be listed below.

\section{Phenomenological analysis}

\subsection{Benchmark points, experimental and theoretical constraints}

In our present analysis, we first fix the flavour-diagonal set of parameters and then we vary the flavour-nondiagonal parameters and explore the bounds on those parameters by theoretical and experimental constraints. We fix the flavour-diagonal parameter set following three very popular SUSY benchmark points, namely SPS1a' [26], $I^{\prime \prime}$ and $\gamma$ [27]. The first two points are mSUGRA points whereas the third one is a mSUGRA point with non-universal Higgs-mass parameters at the GUT-scale. SPS1a' contains the lightest spectrum with squarks around $500 \mathrm{GeV}$ and $m_{\tilde{g}}$ around $600 \mathrm{GeV}, \tan \beta=10$, followed by $\gamma$ with squark masses around $600 \mathrm{GeV}, m_{\tilde{g}}$ around $580 \mathrm{GeV}, \tan \beta=20$, and $I^{\prime \prime}$ with squark masses around $730 \mathrm{GeV}, m_{\tilde{g}}$ around $1000 \mathrm{GeV}, \tan \beta=35$. For more information on the benchmark points see refs. [26, 27]. All of them are consistent with WMAP data [28] and measurements of the anomalous magnetic moment of the muon.

On the flavour-nondiagonal parameter set we pose the following concrete constraints: 
- We explicitly check if all our data points fulfill the theoretical vacuum stability constraints [25]. Actually, all our data points fulfill the conservative condition that in the LR and RL submatrix off-diagonal elements are not larger than diagonal elements, which implies the usual vacuum stability conditions [25].

- Among the constraints from electroweak precision data, the most important one is $m_{h^{0}} \geq 114.4 \mathrm{GeV}$, where we add $3 \mathrm{GeV}$ to the theoretical prediction of $m_{h^{0}}$ as a measure of the theoretical uncertainty [29-31]. Furthermore we require that $M_{W}=$ $80.40 \pm 0.03 \mathrm{GeV}$ where we take as input $m_{Z}=91.187 \mathrm{GeV}, G_{F}=1.16639 \cdot 10^{-5} \mathrm{GeV}^{2}$ and $\alpha_{e m}(0)=1 / 137.0359895$ [32].

- Squark Tevatron bounds on squarks are of the order of $250 \mathrm{GeV}$ depending on the SUSY spectrum [33].

- The explicit experimental constraints from the most important flavour observables we use in our analysis are [34-39]:

$$
\begin{array}{ccc}
2.67<\quad \operatorname{Br}\left(\bar{B} \rightarrow X_{s} \gamma\right) \times 10^{4} & <4.29 \\
13.5<\quad \Delta M_{B_{S}} \text { ps } & <21.1 \\
1.05<B R\left(\bar{B} \rightarrow X_{s} l^{+} l^{-}\right) \text {low } q^{2} \times 10^{6} & <2.15 \\
B R\left(B_{s} \rightarrow \mu^{+} \mu^{-}\right) \times 10^{8} & \leq 5.8
\end{array}
$$

Those bounds include experimental and theoretical errors which are linearly added. Explicitly our bounds are the experimental 95\% bounds where twice the SM error is added in order to take into account uncertainties of the new physics contributions in a conservative way. We have also checked that the recent experimental data on $B \rightarrow \tau \nu$ do not give additional constraints.

- Another requirement is that the lightest stable particle (LSP) should be neutral (but not necessarily be the neutralino) which however is fulfilled once the experimental bounds on the squark masses are taken into account.

For the numerical evaluation we use an updated version of SPheno [40] which has been extended to accept flavour mixing entries in the sfermion mass matrices. The masses and mixings of all SUSY particles are calculated at the one-loop level where the formulas of ref. [41] have been extended for the flavour-mixing case [42]. The masses of the neutral Higgs bosons are calculated at the two-loop level. For the $B$-physics observables we use the formulas of refs. [34, 43-45] for $\bar{B} \rightarrow X_{s} \gamma$, refs. [39, 44-46] for $\bar{B} \rightarrow X_{s} \ell^{+} \ell^{-}$, and ref. [38] for $\Delta M_{B_{s}}$ and $B_{s} \rightarrow \mu^{+} \mu^{-}$. The branching ratios for squark and gluino decays are calculated using tree-level accuracy but with running couplings evaluated at the scale $Q=m$ where $m$ is the mass of the decaying particle. 


\begin{tabular}{|c|c|c|c|}
\hline & SPS1a' & $\gamma$ & $\mathrm{I}^{\prime \prime}$ \\
\hline$\delta_{L L, 23}$ & $(-0.05,0.03)$ & $(-0.037,0.005)$ & $(-0.06,0.001)$ \\
\hline$\delta_{d, R R, 23}$ & $(-0.43,0.66)$ & $(-0.29,0.48)$ & $(-0.5,0.45)$ \\
\hline$\delta_{u, R R, 23}$ & $(-0.7,0.7)$ & $(-0.54,0.43)$ & $(-0.55,0.45)$ \\
\hline$\delta_{u, L R, 23}$ & $(-0.16,0.08)$ & $(-0.16,0.06)$ & $(-0.35,0.05)$ \\
\hline$\delta_{u, L R, 32}$ & $(-0.7,0.54)$ & $(-0.5,0.2)$ & $(-0.7,0.27)$ \\
\hline$\delta_{d, L R, 23}$ & $(-0.0047,0.0046)$ & $(-0.006,0.001)$ & $(-0.01,0.0015)$ \\
\hline$\delta_{d, L R, 32}$ & $(-0.019,0.02)$ & $(-0.015,0.015)$ & $(-0.004,0.003)$ \\
\hline
\end{tabular}

Table 1. Allowed ranges of the $\delta$ parameters in the neighborhood of the benchmark points SPS1a', $\gamma$ and $\mathrm{I}^{\prime \prime}$ taking into account the experimental information given in the text and assuming that only one flavour-mixing parameter is present. The regions correspond to $95 \% \mathrm{CL}$.

\subsection{Results}

\subsubsection{Constraints on flavour parameters}

In our analysis we want to identify the flavour violating decay channels of squarks and the gluino with potentially large branching ratios. And we are interested how the various decays can be combined to obtain information on the parameters.

In table 1 we present the allowed ranges of the flavour-diagonal parameters $\delta_{i j}$ in the $b$ $s$ sector using the so-called 'one-mass-insertion approximation' where just one $\delta$ parameter is assumed to be active and all the others are set to zero. Note that we have calculated observables using the exact diagonalization of the mass matrix of eq. (2.6). Our findings are consistent with the results of previous analyses in refs. [12, 20, 21, 47, 48], in particular the pattern of the constraints on the up-quark sector. Here it turns out that the parameter $\delta_{u, L R, 23}$ receives bounds from the flavour and electroweak constraints while the other two parameters $\delta_{u, L R, 32}$ and $\delta_{u, R R, 23}$ are unconstrained in scenarios with low and moderate $\tan \beta$ and weakly constrainted once $\tan \beta$ gets large.

In figure 1 we present regions in the $\delta_{d, R R, 23}-\delta_{L L, 23}$ (left) and in the $\delta_{d, L R, 23}-\delta_{d, L R, 32}$ planes (right) consistent with experimental data for the SPS1a' benchmark point. We show here the lines corresponding to the constraints from $b \rightarrow s \gamma$ using red (full) lines and $\Delta M_{B_{s}}$ using magenta (dashed) ones. The areas consistent with all constraints correspond to the blue (dark) areas. In both cases the complete plane is compatible also with the constraints due to the observable $b \rightarrow s l^{+} l^{-}, B_{s} \rightarrow \mu^{+} \mu^{-}$and $B_{u} \rightarrow \tau \nu$. One sees the regions allowed by all constraints corresponding to the blue (dark) area are mainly along the axes as one would naively expect. Note, however, that the constraint on a parameter gets more involved once one allows for additional sources of flavour violation. For example, figure 1a shows that for small negative $\delta_{L L, 23}$ the constraint on $\delta_{d, R R, 23}$ becomes first weaker; for larger $\delta_{L L, 23}$ the bound is stronger again.

In both planes of figure 1 we find an additional region consistent with the data in the upper right corner where both flavour mixing parameters are sizable. However, in these regions clearly some cancellations between various contributions to the observable take place. In particular the gluino and the chargino contributions for $\Delta M_{B_{s}}$ are large and of opposite sign. Their modulus can reach about twice the size of the SM contribution. 
a)

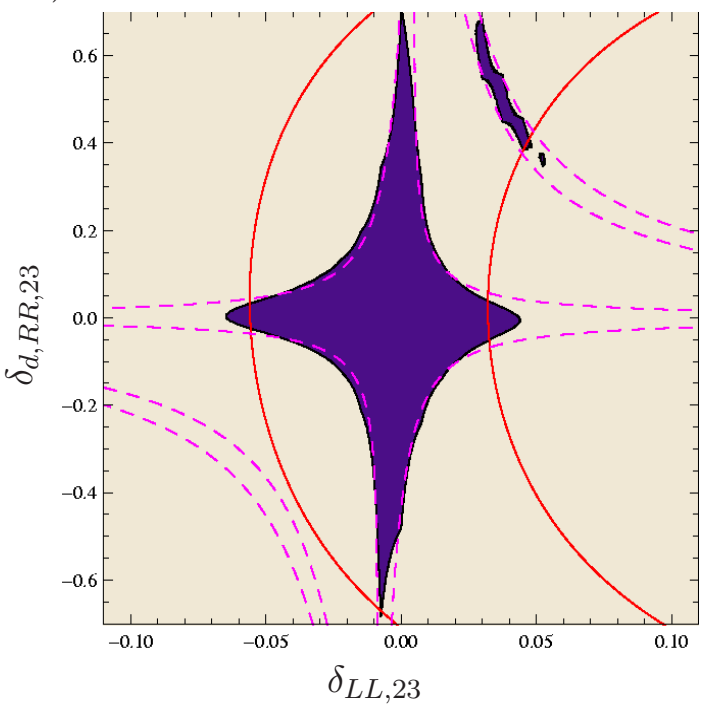

b)

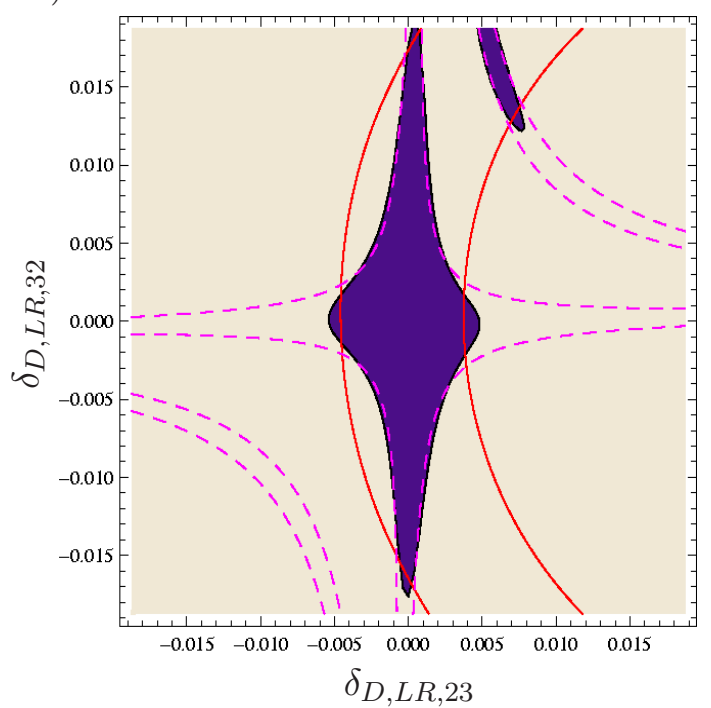

Figure 1. Allowed regions a) in the $\delta_{d, R R, 23}-\delta_{L L, 23}$ and b) in the $\delta_{D, L R, 23}-\delta_{D, L R, 32}$ plane for SPS1a'. All other than the shown flavour off-diagonal elements are put to zero. The lines correspond to: full red lines $b \rightarrow s \gamma, 2.6710^{-4}$ and $, b \rightarrow s \gamma, 4.2910^{-4}$, dashed magenta lines $\left|\Delta_{M_{B_{s}}}\right|=13.5 \mathrm{ps}^{-1}$, and $\left|\Delta_{M_{B_{s}}}\right|=21.1 \mathrm{ps}^{-1}$. The blue (dark) area shows the regions consistent with all data at $95 \%$ CL.

The other two benchmark points show very similar features but for the fact that in case of $I^{\prime \prime}$ also $B_{s} \rightarrow \mu^{+} \mu^{-}$would exclude part of the allowed regions.

\subsubsection{Flavour violating decays of squarks and gluinos}

In the following we discuss the effect of the flavour mixing parameters on the decay properties of squarks and gluinos. For the discussion of the basic features we will first take two points in the allowed region of figure 1a). Afterwards we discuss various parameter dependencies. Note that we often show only part of the kinematically accessible final states and, thus, the branching ratios shown do not sum necessarily up to 1 . For the flavour diagonal entries we will use the point SPS1a' but again similar features are found for the other benchmark points as well. The main difference is due to the kinematics.

The two study points chosen are characterized by $\delta_{L L, 23}=0.01$ and $\delta_{D, R R 23}=0.1$ (point I) and $\delta_{L L, 23}=0.04$ and $\delta_{D, R R 23}=0.45$ (point II) respectively. Study point II is characterized by large cancellations of the SUSY contributions to $B$-physics observables. In table 2 we give a summary of the various branching ratios and in table 3 we display the masses. For comparison also the masses without flavour mixing parameters are given.

The first feature to note is that the bounds on $\delta_{L L}$ are already so strong that there are small effects only on masses or branching ratios due to this flavour-mixing parameter. ${ }^{2}$ This implies that in our examples the masses and branching ratios of the $u$-type squarks are hardly altered compared to the SPS1a' point. Things are different for the $d$-type squarks and consequently also for the gluino as the $\delta_{d, R R, 23}$ parameter can be sizable.

\footnotetext{
${ }^{2}$ Note that this strong constraint would be weakened if more than two deltas are active as shown in the study point given in ref. [11] that contains such an example.
} 


\begin{tabular}{|c|r|r|r|r|r|r|}
\hline decaying & \multicolumn{6}{|c|}{ final states and corresponding branching ratios in \% for. } \\
particle & \multicolumn{2}{|c|}{ I. $\delta_{L L, 23}=0.01, \delta_{D, R R 23}=0.1$} & \multicolumn{2}{|c|}{ II. $\delta_{L L, 23}=0.04, \delta_{D, R R 23}=0.45$} \\
\hline$\tilde{d}_{1} \rightarrow$ & $\tilde{\chi}_{1}^{0} b, 4.4$ & $\tilde{\chi}_{2}^{0} b, 29.8$ & $\tilde{\chi}_{1}^{-} t, 37.0$ & $\tilde{\chi}_{1}^{0} s, 36.8$ & $\tilde{\chi}_{1}^{0} b, 42.2$ & $\tilde{\chi}_{2}^{0} b, 10.9$ \\
& $\tilde{u}_{1} W^{-}, 27.7$ & & & $\tilde{\chi}_{1}^{-} t, 9.6$ & & \\
\hline$\tilde{d}_{2} \rightarrow$ & $\tilde{\chi}_{1}^{0} s, 8.0$ & $\tilde{\chi}_{1}^{0} b, 6.4$ & $\tilde{\chi}_{2}^{0} b, 19.0$ & $\tilde{\chi}_{1}^{0} b, 2.1$ & $\tilde{\chi}_{2}^{0} b, 27.3$ & $\tilde{\chi}_{1}^{-} t, 34.6$ \\
& $\tilde{\chi}_{3}^{0} b, 1.1$ & $\tilde{\chi}_{4}^{0} b, 1.8$ & $\tilde{\chi}_{1}^{-} t, 24.6$ & $\tilde{u}_{1} W^{-}, 33.2$ & & \\
& $\tilde{u}_{1} W^{-}, 38.9$ & & & & & \\
\hline$\tilde{d}_{4} \rightarrow$ & $\tilde{\chi}_{1}^{0} s, 9.1$ & $\tilde{\chi}_{1}^{0} b, 6.3$ & $\tilde{\chi}_{2}^{0} s, 25.3$ & $\tilde{\chi}_{1}^{0} d, 2.3$ & $\tilde{\chi}_{2}^{0} d, 31.7$ & $\tilde{\chi}_{1}^{-} u, 59.7$ \\
& $\tilde{\chi}_{1}^{-} u, 2.1$ & $\tilde{\chi}_{1}^{-} c, 47.3$ & $\tilde{u}_{1} W^{-}, 4.8$ & $\tilde{\chi}_{1}^{-} c, 3.0$ & $\tilde{\chi}_{2}^{-} u, 2.3$ & \\
\hline$\tilde{d}_{5} \rightarrow$ & $\tilde{\chi}_{1}^{0} d, 2.3$ & $\tilde{\chi}_{2}^{0} d, 31.7$ & $\tilde{\chi}_{1}^{-} u, 59.9$ & $\tilde{\chi}_{1}^{0} s, 2.2$ & $\tilde{\chi}_{2}^{0} s, 30.7$ & $\tilde{\chi}_{1}^{-} u, 2.9$ \\
& $\tilde{\chi}_{1}^{-} c, 2.8$ & $\tilde{\chi}_{2}^{-} u, 2.3$ & & $\tilde{\chi}_{1}^{-} c, 58.5$ & $\tilde{\chi}_{2}^{-} c, 2.3$ & \\
\hline$\tilde{d}_{6} \rightarrow$ & $\tilde{\chi}_{1}^{0} s, 3.1$ & $\tilde{\chi}_{2}^{0} s, 30.6$ & $\tilde{\chi}_{1}^{-} u, 2.7$ & $\tilde{\chi}_{1}^{0} s, 19.7$ & $\tilde{\chi}_{1}^{0} b, 18.8$ & $\tilde{\chi}_{3}^{0} b, 2.9$ \\
& $\tilde{\chi}_{1}^{-} c, 58.1$ & $\tilde{\chi}_{2}^{-} c, 2.4$ & & $\tilde{\chi}_{4}^{0} b, 2.9$ & $\tilde{\chi}_{2}^{-} t, 5.8$ & $\tilde{g}^{-} s, 2.2$ \\
& & & & $\tilde{g}^{2} b, 39.8$ & $\tilde{u}_{1} W^{-}, 5.5$ & \\
\hline$\tilde{g}^{\prime} \rightarrow$ & $\tilde{u}_{1} t, 19.2$ & $\tilde{u}_{2} c, 8.2$ & $\tilde{u}_{3} u, 8.3$ & $\tilde{u}_{1} t, 13.5$ & $\tilde{u}_{2} c, 5.8$ & $\tilde{u}_{3} u, 5.8$ \\
& $\tilde{u}_{4} u, 4.2$ & $\tilde{u}_{5} c, 4.2$ & & $\tilde{u}_{4} c, 2.6$ & $\tilde{u}_{5} u, 2.6$ & \\
& $\tilde{d}_{1} s, 1.4$ & $\tilde{d}_{1} b, 20.6$ & & $\tilde{d}_{1} s, 21.1$ & $\tilde{d}_{1} b, 22.7$ & \\
& $\tilde{d}_{2} s, 6.3$ & $\tilde{d}_{2} b, 9.0$ & $\tilde{d}_{3} d, 8.3$ & $\tilde{d}_{2} b, 14.0$ & & $\tilde{d}_{3} d, 5.9$ \\
& $\tilde{d}_{4} s, 2.3$ & $\tilde{d}_{4} b, 1.3$ & $\tilde{d}_{6} s, 2.8$ & $\tilde{d}_{4} d, 2.3$ & $\tilde{d}_{5} d, 3.3$ & \\
\hline
\end{tabular}

Table 2. Branching ratios larger than $1 \%$ for two study points. The flavour diagonal entries are according to SPS1a'. $\tilde{u}_{i}$ decays are like in SPS1a' [26] and in both scenarios $\operatorname{BR}\left(\tilde{d}_{3} \rightarrow \tilde{\chi}_{1}^{0} d\right)=99.1 \%$.

\begin{tabular}{|c|c|c|c|c|c|c|c|c|c|c|}
\hline & $m_{\tilde{d}_{1}}$ & $m_{\tilde{d}_{2}}$ & $m_{\tilde{d}_{3}}$ & $m_{\tilde{d}_{4}}$ & $m_{\tilde{d}_{5}}$ & $m_{\tilde{d}_{6}}$ & $m_{\tilde{u}_{1}}$ & $m_{\tilde{u}_{2}}$ & $m_{\tilde{u}_{4}}$ & $m_{\tilde{u}_{6}}$ \\
SPS1a' & 506 & 546 & 547 & 547 & 570 & 570 & 367 & 547 & 565 & 586 \\
I. $\delta_{L L, 23}=0.01, \delta_{d, R R, 23}=0.1$ & 503 & 525 & 547 & 569 & 570 & 570 & 366 & 547 & 565 & 586 \\
II. $\delta_{L L, 23}=0.04, \delta_{d, R R, 23}=0.45$ & 422 & 509 & 547 & 570 & 572 & 641 & 366 & 547 & 565 & 587 \\
\hline
\end{tabular}

Table 3. Squark masses in GeV for SPS1a' and our two points under study. The flavour diagonal entries are according to SPS1a'. Note that $m_{\tilde{u}_{2}} \simeq m_{\tilde{u}_{3}}$ and $m_{\tilde{u}_{4}} \simeq m_{\tilde{u}_{5}}$.

The relative size of the branching ratios in table 2 can be understood by the nature of the various squarks mass eigenstates. In point I one finds $\tilde{d}_{1} \simeq \tilde{b}_{L}$ with a small admixture of $\tilde{b}_{R}, \tilde{d}_{2} \simeq \tilde{b}_{R}$ with small admixtures of $\tilde{s}_{R}$ and $\tilde{b}_{L}, \tilde{d}_{3} \simeq \tilde{d}_{R}, \tilde{d}_{4} \simeq \tilde{s}_{R}$ with admixtures of $\tilde{s}_{L}$ and $\tilde{b}_{R}, \tilde{d}_{5} \simeq \tilde{d}_{L}$ and $\tilde{d}_{6} \simeq \tilde{s}_{L}$ with a small admixture of $\tilde{s}_{R}$. Thus, larger flavour effects are visible in the decays of $\tilde{d}_{2}$ and $\tilde{d}_{4}$ where the flavour violating decay branchings ratios $\tilde{d}_{2} \rightarrow \tilde{\chi}_{1}^{0} s$ and $\tilde{d}_{4} \rightarrow \tilde{\chi}_{1}^{0} b$ are of the order of $10 \%$. This structure is also the reason for the relative importance of the flavour violating decays of the gluino. As a side remark we note that the flavour violating decays of the first generation squarks and of the $2 \mathrm{nd} / 3 \mathrm{rd}$ generation squarks into the first generation quarks are due to CKM quark mixing.

In point II the situation is more complicated due to the larger flavour mixing parameters. With respect to the nature of the $d$-type squarks we find that $\tilde{d}_{1}$ and $\tilde{d}_{6}$ are strongly mixed states consisting mainly of $\tilde{s}_{R}$ and $\tilde{b}_{R}$ with a small admixture of $\tilde{b}_{L}$ whereas the other states are mainly electroweak eigenstates: $\tilde{d}_{2} \simeq \tilde{b}_{L}, \tilde{d}_{3} \simeq \tilde{d}_{R}, \tilde{d}_{4} \simeq \tilde{d}_{L}$ and $\tilde{d}_{5} \simeq \tilde{s}_{L}$. In this 
a)

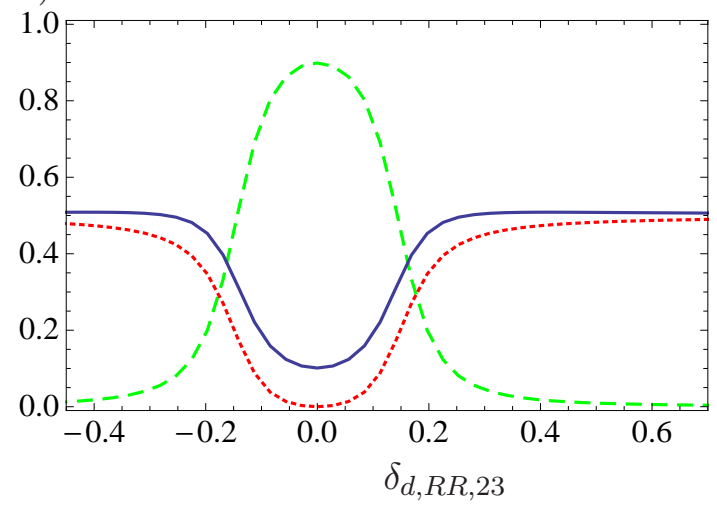

b)

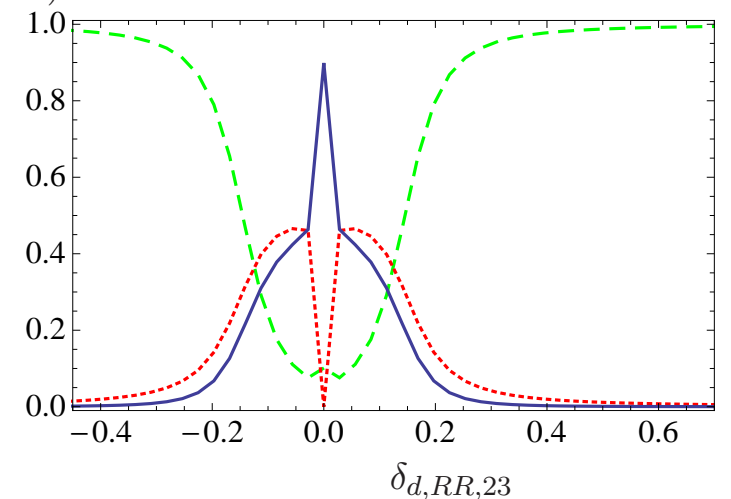

Figure 2. Composition of a) $\tilde{d}_{i=1}$ and b) $\tilde{d}_{i=2}$ as a function of $\delta_{d, R R, 23}$, the flavour diagonal parameters are the ones of SPS1a'. The flavour basis is given by $\left(\tilde{d}_{L}, \tilde{s}_{L}, \tilde{b}_{L}, \tilde{d}_{R}, \tilde{s}_{R}, \tilde{b}_{R}\right)$. The lines correspond to the final states: dashed green line to $\left|R_{i, \tilde{b}_{L}}^{d}\right|^{2}$, dotted red line to $\left|R_{i, \tilde{s}_{R}}^{d}\right|^{2}$, and full blue line to $\left|R_{i, \tilde{b}_{R}}^{d}\right|^{2}$.

scenario the flavour violating final states can even reach about $40 \%$ in case of $\tilde{d}_{1} \rightarrow \tilde{\chi}_{1}^{0} s$ and about $20 \%$ for $\tilde{d}_{6} \rightarrow \tilde{\chi}_{1}^{0} b$. The differences for the gluino decays between these two points is not only due to the different mixing in the $d$-squark sector but also due to the different kinematics as can be seen from table 3 .

The effect of the flavour mixing parameters on gluino decays is strong except for $\delta_{d, L R, i j}$. We first investigate how the nature of the various squark states change when varying the flavour mixing entries. In figure 2 we display the composition of the two lightest $d$-squarks as a function of $\delta_{d, R R, 23}$. The lightest state is mainly a $\tilde{b}_{L}$ state for $\left|\delta_{d, R R, 23}\right| \lesssim 0.15$. At $\left|\delta_{d, R R, 23}\right| \lesssim 0.2$ it is a strongly mixed state consisting of $\tilde{b}_{L}, \tilde{b}_{R}$ and $\tilde{s}_{R}$ whereas for larger values of $\left|\delta_{d, R R, 23}\right|$ it is mainly an admixture of $\tilde{b}_{R}$ and $\tilde{s}_{R}$. The second state is mainly a $\tilde{b}_{R}$ state for $\left|\delta_{d, R R, 23}\right| \lesssim 0.05$. The kinks around 0.05 stem from the fact that the flavour mixing is about as large as the left-right mixing in the sbottom sector. Around $\left|\delta_{d, R R, 23}\right| \lesssim 0.15$ the $\tilde{b}_{L}$ component starts to dominate; for $\left|\delta_{d, R R, 23}\right| \gtrsim 0.3$ this state is almost a pure $\tilde{b}_{L}$.

This behaviour is reflected in the gluino decays as can be seen in figure 3 where we show the gluino decay branching ratios into the two lightest $d$-squarks and into the two lightest $u$-squarks as a function of $\delta_{d, R R, 23}$. As already indicated in the discussion above, the dependence is twofold. The larger the off-diagonal elements are, the larger is the corresponding flavour violating coupling; but also kinematical effects play an important rôle, e.g. the larger $\delta_{d, R R, 23}$ is, the lighter $\tilde{d}_{1}$ gets leading to an increased phase space. The kinks and the crossing of the various lines correspond exactly to the changes in the nature of the $d$-squarks. Figure $3 \mathrm{c}$ shows in addition that there are scenarios where one can have sizeable flavour violating decays into $d$-squarks and into $u$-squarks at the same time.

Similar features can be observed for the case of large flavour mixing in the $u$-squark sector. In figure 4 we display the flavour content of the two lightest $u$-squarks as a function of $\delta_{u, R R, 23}$. There are qualitative differences compared to the $d$-squarks because (i) the left-right mixing in the top-squark sector is significantly large and (ii) the lightest state $\tilde{u}_{1}$ 
a)

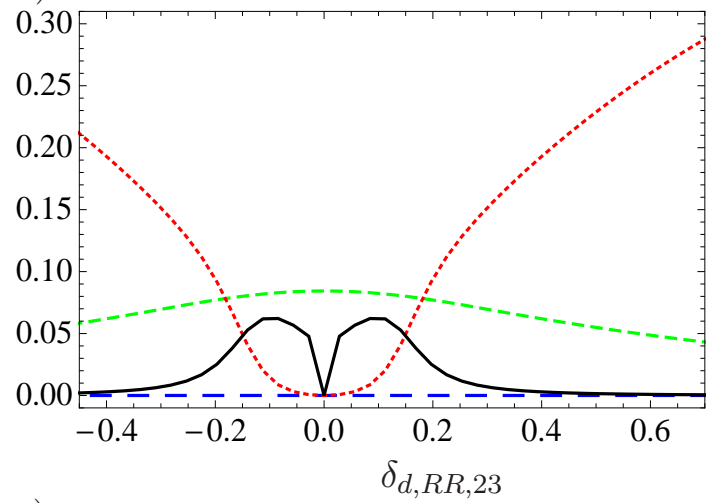

c)

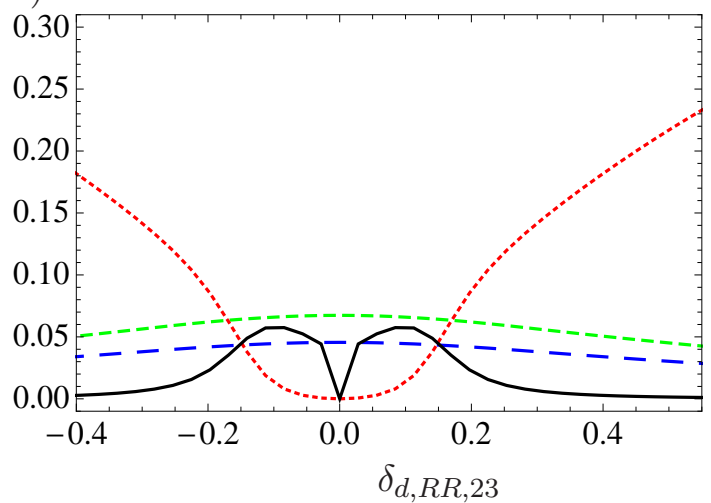

b)

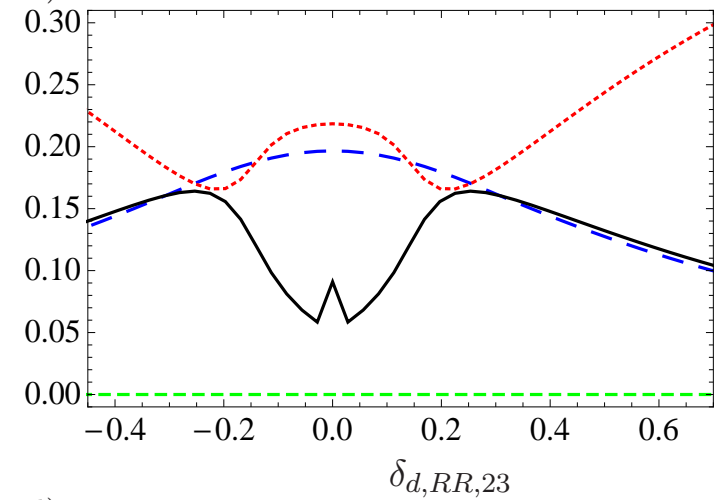

d)

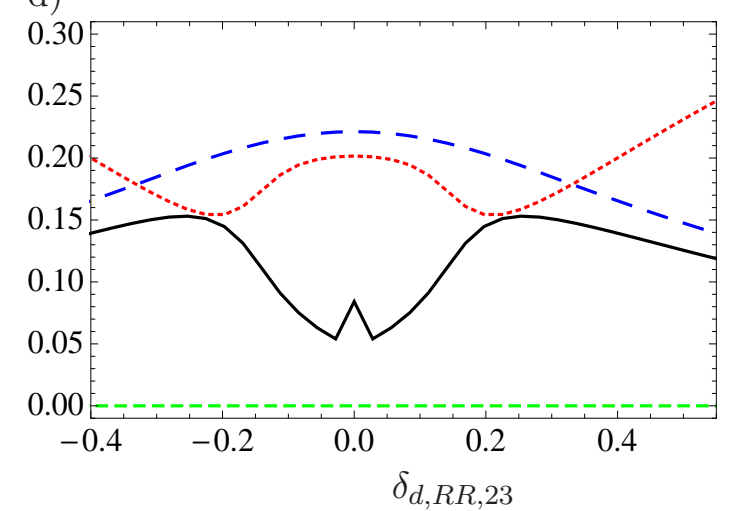

Figure 3. $\tilde{g}$ decays as a function of $\delta_{d, R R, 23}$, in a) and b) all other $\delta$ s are zero whereas in c) and d) $\delta_{u, R R, 23}=0.2$ in addition but all other $\delta$ parameter are zero. The lines in a) and c) correspond to the final states $\tilde{u}_{1} c$ (long dashed blue line), $\tilde{u}_{2} c$ (short dashed green line), $\tilde{d}_{1} s$ (dotted red line), and $\tilde{d}_{2} s$ (full black line); the lines in b) and d) correspond to the final states $\tilde{u}_{1} t$ (long dashed blue line), $\tilde{u}_{2} t$ (short dashed green line), $\tilde{d}_{1} b$ (dotted red line), and $\tilde{d}_{2} b$ (full black line).

a)

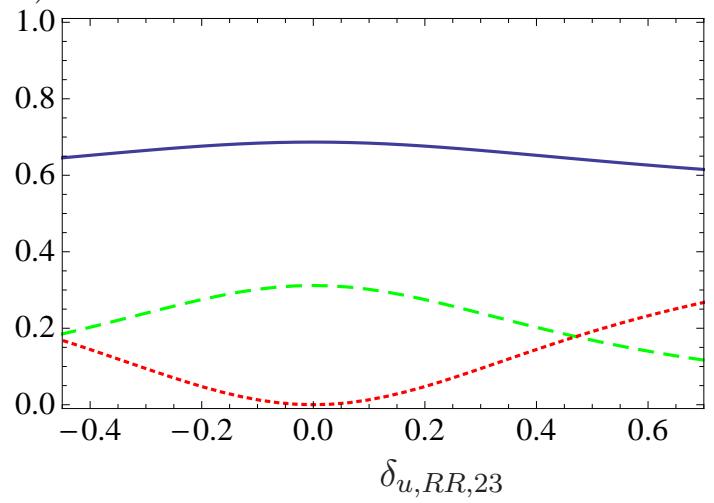

b)

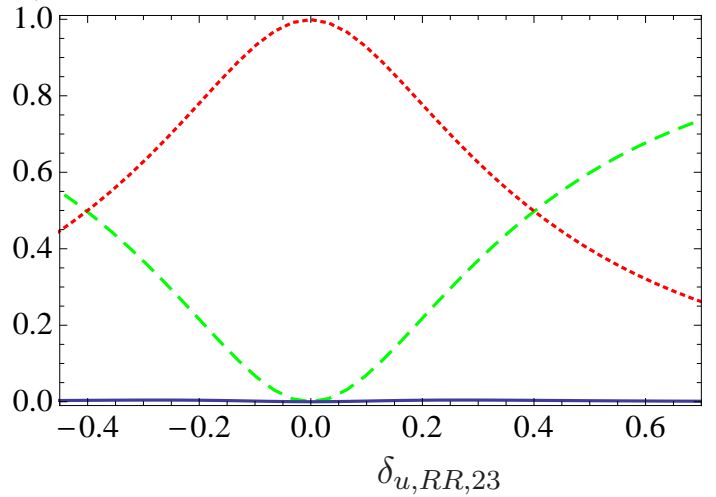

Figure 4. Composition of a) $\tilde{u}_{i=1}$ and b) $\tilde{u}_{i=2}$ as a function of $\delta_{u, R R, 23}$, the flavour diagonal parameters are the ones of SPS1a'. The flavour basis is given by $\left(\tilde{u}_{L}, \tilde{c}_{L}, \tilde{t}_{L}, \tilde{u}_{R}, \tilde{c}_{R}, \tilde{t}_{R}\right)$. The lines correspond to the following final states: dashed green line to $\left|R_{i, \tilde{t}_{L}}^{u}\right|^{2}$, dotted red line to $\left|R_{i, \tilde{c}_{R}}^{u}\right|^{2}$, and full blue line to $\left|R_{i, \tilde{t}_{R}}^{u}\right|^{2}$. 
a)

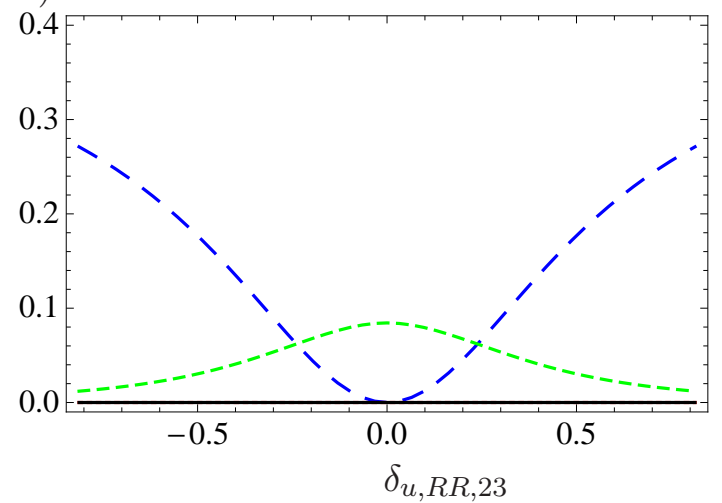

b)

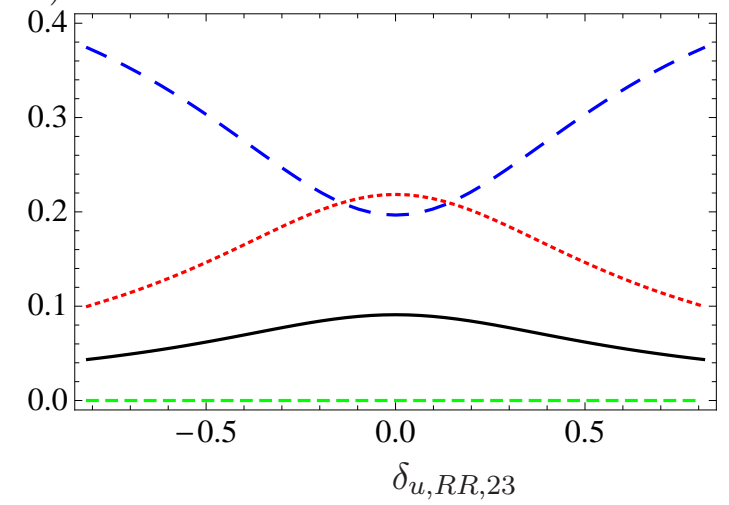

Figure 5. $\tilde{g}$ decays as a function of $\delta_{u, R R, 23}$ and all other $\delta$ parameters are zero. The lines in a) correspond to the final states $\tilde{u}_{1} c$ (long dashed blue line), $\tilde{u}_{2} c$ (short dashed green), and $\tilde{d}_{2} s$ (full black); the lines in b) correspond to the final states $\tilde{u}_{1} t$ (long dashed), $\tilde{u}_{2} t$ (short dashed green), $\tilde{d}_{1} b$ (dotted red), and $\tilde{d}_{2} b$ (full black).

in the flavour conserving case is a mixture of $\tilde{t}_{R}$ and $\tilde{t}_{L}$ and $\tilde{u}_{2} \simeq \tilde{c}_{R}$. We see that with increasing $\delta_{u, R R, 23}$ not only the mixing between $\tilde{t}_{R}$ and $\tilde{c}_{R}$ get larger as expected but that at the same time the admixture of $\tilde{t}_{L}$ decreases quickly. The second state is not, as one would naively expect, a strongly mixed state consisting of $\tilde{t}_{R}$ and $\tilde{c}_{R}$ but a mixture between $\tilde{t}_{L}$ and $\tilde{c}_{R}$ and only a negligible admixture of $\tilde{t}_{R}$. The reason for this surprising feature is that the left-right mixing in the top-squark sector is larger than $\delta_{u, R R, 23}$ for the complete range shown.

Again this is reflected in the gluino decays shown in figure 5 where we display the gluino decay branching ratios as a function of $\delta_{u, R R, 23}$. Here the main flavour effect is the increase of the branching ratio into $\tilde{u}_{1} c$ (long dashed blue line on the left side). The expected increase of $\tilde{u}_{2} t$ does not take place due to kinematics as $m_{\tilde{u}_{2}}+m_{t}>m_{\tilde{g}}$ for most of the range shown. One would expect that the branching ratio for $\tilde{u}_{1} t$ should decrease for increasing $\left|\delta_{u, R R, 23}\right|$ as the top-squark components decrease. However, this decrease is overcompensated by the increased kinematics as the mass of $\tilde{u}_{1}$ drops to a value about $200 \mathrm{GeV}$.

The squarks stemming from the gluino decays will decay further and in figures 6 and 7 we display the corresponding branching ratios of the lightest $u$-squark and $d$-squark, respectively. The behaviour of $\tilde{u}_{1}$ decays in figure 6 is a direct consequence of the above described change of its nature with increasing $\left|\delta_{u, R R, 23}\right|$. But also its mass decrease again implies that if $\left|\delta_{u, R R, 23}\right|$ increases first the final state $\tilde{\chi}_{2}^{0} t$ and then also the final states $\tilde{\chi}_{1}^{+} b$ and $\tilde{\chi}_{1}^{0} t$ get kinematically forbidden. The kinks in the various lines are a direct consequence of these kinematical effects. Finally, for $\left|\delta_{u, R R, 23}\right| \gtrsim 0.6$ only the final state $\tilde{\chi}_{1}^{0} c$ is possible. We have checked that three and four body decay modes, such as $b W \tilde{\chi}_{1}^{0}[49], \tilde{l} \nu b[50,51]$ or $b f \bar{f}^{\prime} \tilde{\chi}_{1}^{0}[52]$, are suppressed and have branching ratios below $1 \%$.

In figure 7 we display the branching ratios of $\tilde{d}_{1}$. The only sizeable flavour violating decay channel is $\tilde{\chi}_{1}^{0} s$ whereas $\tilde{\chi}_{1}^{-} c$ is suppressed because we consider here only flavour mixing between the $R$-squarks. The above mentioned level crossing at $\left|\delta_{d, R R, 23}\right| \simeq 0.15$ is the main reason for the drop of the final states $\tilde{\chi}_{2}^{0} b$ and $\tilde{\chi}_{1}^{-} t$ besides kinematical effects. 

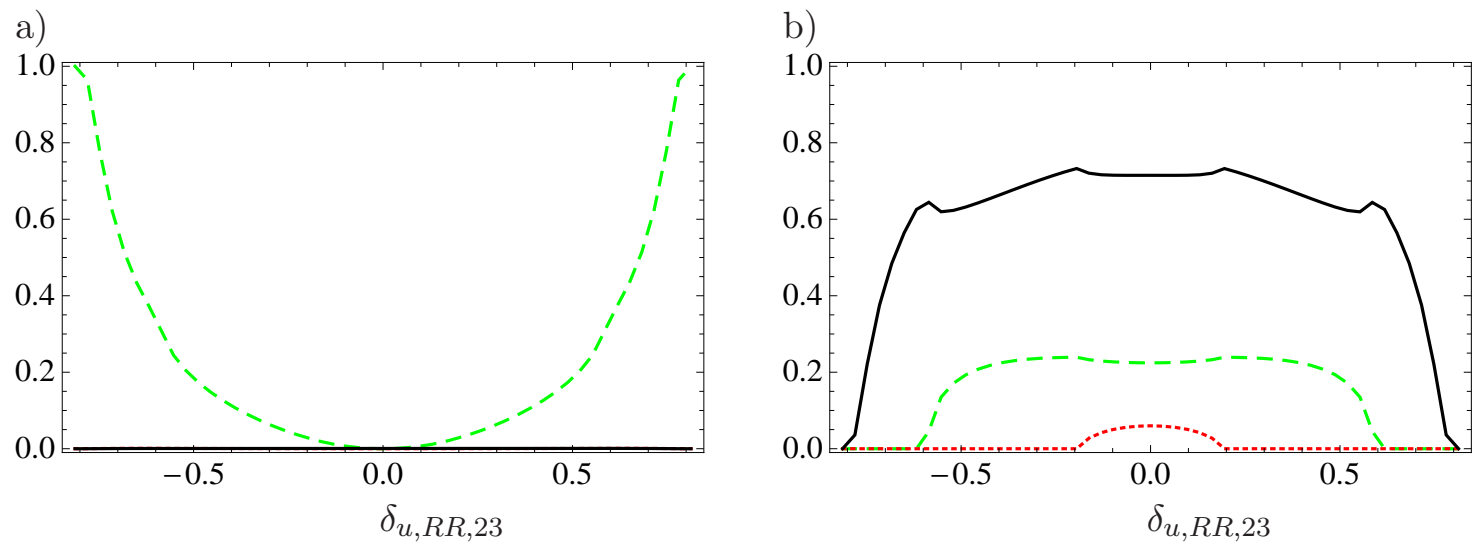

Figure 6. $\tilde{u}_{1}$ decays as a function of $\delta_{u, R R, 23}$, the flavour diagonal parameters are the ones of SPS1a'. The lines in a) correspond to the final states $\tilde{\chi}_{1}^{0} c$ (dashed green line), $\tilde{\chi}_{2}^{0} c$ (dotted red), and $\tilde{\chi}_{1}^{+} s$ (full black); the lines in b) correspond to the final states $\tilde{\chi}_{1}^{0} t$ (dashed green), $\tilde{\chi}_{2}^{0} t$ (dotted red), and $\tilde{\chi}_{1}^{+} b$. (full black).

a)

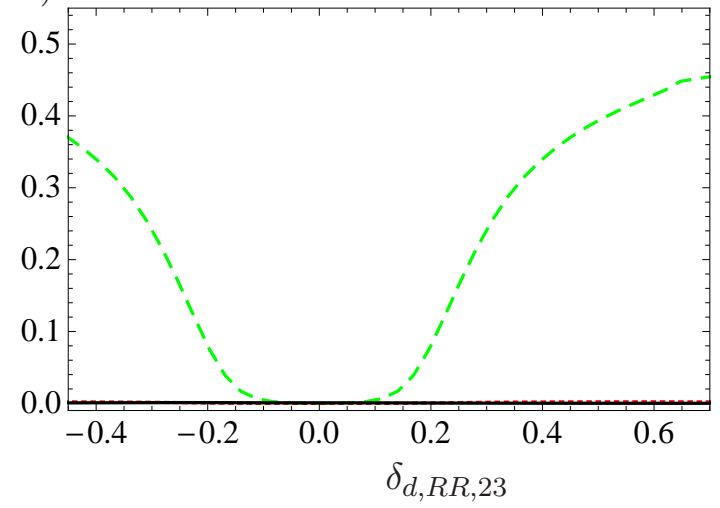

b)

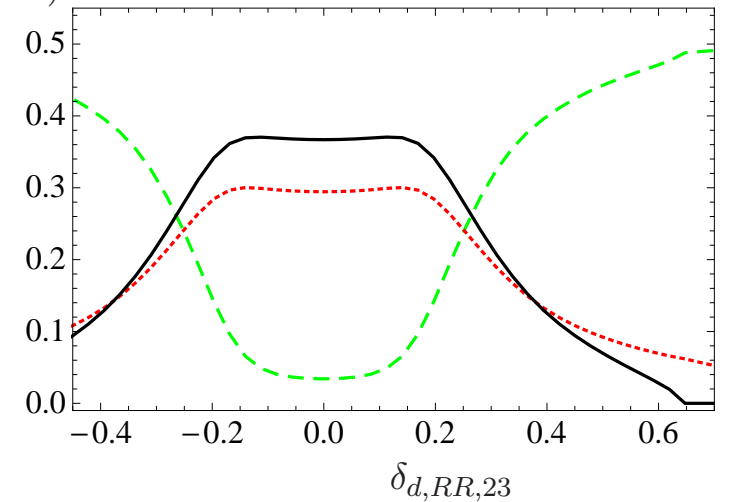

Figure 7. $\tilde{d}_{1}$ decays as a function of of $\delta_{d, R R, 23}$, the flavour diagonal parameters are the ones of SPS1a'. The lines in a) correspond to the final states $\tilde{\chi}_{1}^{0} s$ (dashed green line), $\tilde{\chi}_{2}^{0} s$ (dotted red), and $\tilde{\chi}_{1}^{-} c$ (full black); the lines in b) correspond to the final states $\tilde{\chi}_{1}^{0} b$ (dashed green), $\tilde{\chi}_{2}^{0} b$ (dotted red), and $\tilde{\chi}_{1}^{-} t$ (full black).

Finally, we note that in the case of the benchmark point $\gamma$ we find similar features for the gluino decays into the two lightest squarks. The fact that the other squarks are heavier than the gluino has no large impact, in particular their decay modes into the gluino are small due to the small mass differences between the heavy squarks and the gluino.

\section{Impact for LHC and ILC}

We have seen in figure 1 that constraints due to low-energy observables lead to interesting structures in the parameter space and the only certain parameter combinations are allowed in the case one varies two parameters independently. Of course things are much more complicated once all off-diagonal elements are allowed to be non-zero at the same time. In the end the crucial question is how information on flavour violating squark and gluino decays can be used to pin down the underlying parameters. The answer to this question requires detailed information on masses, production cross sections and branching ratios. 
a) $10^{4} d\left(\operatorname{BR}\left(\tilde{g} \rightarrow b \bar{b} \tilde{\chi}_{1}^{0}\right)\right) / d m_{b b}$

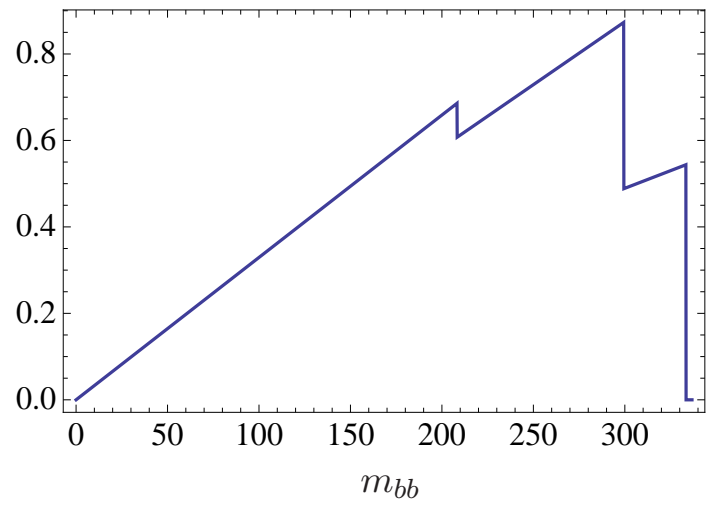

b) $10^{4} d\left(\operatorname{BR}\left(\tilde{g} \rightarrow b s \tilde{\chi}_{1}^{0}\right)\right) / d m_{b s}$

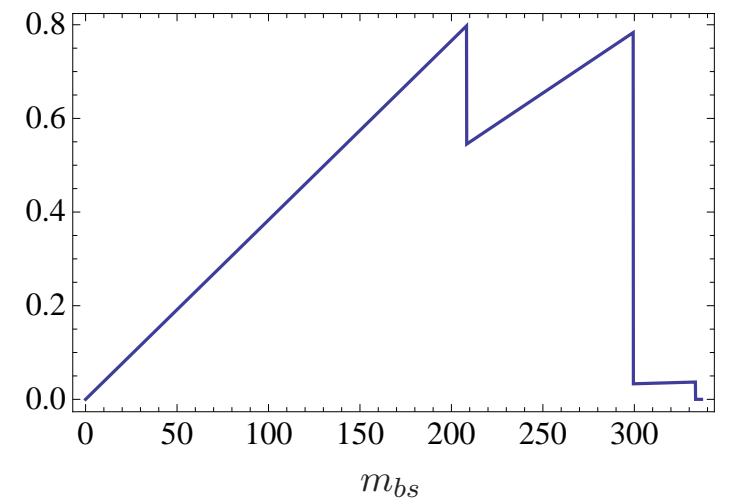

Figure 8. Differential distributions $d\left(\operatorname{BR}\left(\tilde{g} \rightarrow b \bar{b} \tilde{\chi}_{1}^{0}\right)\right) / d m_{b b}$ and $d\left(\operatorname{BR}\left(\tilde{g} \rightarrow b \bar{b} \tilde{\chi}_{1}^{0}\right)\right) / d m_{b b}$ as a function of $m_{b b}=\sqrt{\left(p_{b}+p_{\bar{b}}\right)^{2}}\left(m_{b s}\right)$ for point 1 defined in section 3.2.2. In b) the sum over the charges is shown: $\operatorname{BR}\left(\tilde{g} \rightarrow b \bar{s} \tilde{\chi}_{1}^{0}\right)+\operatorname{BR}\left(\tilde{g} \rightarrow \bar{b} s \tilde{\chi}_{1}^{0}\right)$.

We have seen that squarks and gluinos can have flavour changing decay modes of typically $O(10) \%$ branching ratio. This clearly has an impact on the discovery strategy of squarks and gluinos as well as on the measurement of the underlying parameters at the LHC and a future international linear collider (ILC). For example, in mSUGRA points without flavour mixing one finds usually that the left-squarks of the first two generations as well as the right squarks have similar masses. Large flavour mixing implies that there is a considerable mass splitting as can be seen in table 3 . Therefore, the assumption of almost degenerate masses should be reconsidered if sizable flavour changing decays are discovered in squark and gluino decays.

An important part of the decay chains considered for SPS1a' and nearby points are $\tilde{g} \rightarrow b \tilde{b}_{j} \rightarrow b \bar{b} \tilde{\chi}_{k}^{0}$ which are used to determine the gluino mass as well as the sbottom masses or at least their average value if these masses are close [53].

In the latter analysis the existence of two $b$-jets has been assumed stemming from this decay chain. In this case the two contributing sbottoms would lead to two edges in the partial distribution $d\left(\operatorname{BR}\left(\tilde{g} \rightarrow b \bar{b} \tilde{\chi}_{1}^{0}\right) / d m_{b b}\right.$ where $m_{b b}$ is the invariant mass of the two bottom quarks. As can be seen from figure 8 there are scenarios where more squarks can contribute and consequently one finds a richer structure, e.g. three edges in the example shown corresponding to study point I. Such a structure is either a clear sign of flavour violation or the fact that the particle content of the MSSM needs to be extended. Moreover, also the differential distribution of the final state $b s \tilde{\chi}_{1}^{0}$ shows a similar structure where the edges occur at the same places as in the $b \bar{b}$ spectrum but with different relative heights. This gives a non-trivial cross-check on the hypothesis of sizeable flavour mixing. Clearly a detailed Monte Carlo study will be necessary to see with which precision one can extract information on these edges. However, such a study is well beyond the scope of this paper. Obvious difficulties will be combinatorics because in general two gluinos or a gluino together with a squark will be produced and, thus, there will be several jets stemming from light quarks. However, one could take final states where one gluino decays into $d$-type squarks and the second into stops or $c$-squarks. In the second case effective charm tagging would be a crucial. 
Similar conclusions hold for the decay chains $\tilde{g} \rightarrow c \tilde{u}_{i} \rightarrow c t \tilde{\chi}_{1}^{0}$ and $\tilde{g} \rightarrow t \tilde{u}_{i} \rightarrow c t \tilde{\chi}_{1}^{0}$ analyzed in [54] and also for the the variable $M_{t b}^{w}$ defined in [55] which sums up final states containing $t b \tilde{\chi}_{1}^{+}$.

At an ILC the situation should be considerable easier: first one can tune the center of mass energy so that one studies in principle the states one after each other. Secondly, one can polarize both, electrons and positrons, $[56,57]$ and thus one influences the production rates for signal and background. However, the ILC will be limited to energies in the one $\mathrm{TeV}$ range and, thus, most likely only part of the squarks can be explored at this machine. However, already this partial information combined with LHC data can give useful information [58]. Additional information can then be obtained at a multi-TeV $e^{+} e^{-}$ collider such as CLIC [59].

Up to now we have discussed scenarios with a neutralino LSP. It might well be that either a gravitino $\tilde{G}$ or an axino $\tilde{a}$ is the LSP. In this case one has to take into account the additional decays of the lightest neutralino into the LSP, e.g. $\tilde{\chi}_{1}^{0} \rightarrow \gamma \tilde{G}$. As long as these additional decays do not contain quarks they will have no impact on the signals discussed above.

\section{Conclusions}

Flavour-violating low- and high-energy observables are governed by the same parameters in supersymmetric models. A particular important question is whether the soft SUSY breaking parameters can have additional flavour structures beside the well-known CKM structure. In this paper, we have analysed flavour-violating squark and gluino decays in view of the present flavour data and have shown that they can be typically of order of $10 \%$ in the regions of parameter space where no or only moderate cancellations between different contributions to the low energy observables occur. If we allow for larger new physics contributions, e.g. the same order as the SM contributions, in the flavour observables, then even flavour-violating branching ratios of up to $40 \%$ are consistent with the present data. We have checked that this is a common feature for a couple of SUSY benchmark points like SPS1a', $\gamma$, and $I^{\prime \prime}$. We have explicitly derived the pattern of flavour-violating decay modes from the specific structure of the flavour-violating parameters including kinematical constraints. Finally, we have briefly analyzed the direct consequences for the search of supersymmetric particles in specific examples. The full exploitation of the necessary modifications in the particle search calls for detailed Monte-Carlo analyses.

\section{Acknowledgments}

This work is supported by the European Network MRTN-CT-2006-035505 'HEPTOOLS'. W.P. is is partially supported by the DFG, project Nr. PO 1337/1-1.

\section{References}

[1] T. Hurth, Status of SM calculations of $b \rightarrow s$ transitions, Int. J. Mod. Phys. A 22 (2007) 1781 [hep-ph/0703226] [SPIRES]. 
[2] T. Hurth, Present status of inclusive rare B decays, Rev. Mod. Phys. 75 (2003) 1159 [hep-ph/0212304] [SPIRES].

[3] CMS collaboration, G.L. Bayatian et al., CMS technical design report. Volume II: physics performance, J. Phys. G 34 (2007) 995 [SPIRES].

[4] F. del Aguila et al., Collider aspects of flavour physics at high $Q$, Eur. Phys. J. C 57 (2008) 183 [arXiv:0801.1800] [SPIRES].

[5] M. Artuso et al., B, D and $K$ decays, Eur. Phys. J. C 57 (2008) 309 [arXiv:0801.1833] [SPIRES].

[6] The ATLAS collaboration, G. Aad et al., Expected Performance of the ATLAS Experiment - Detector, Trigger and Physics, arXiv:0901.0512 [SPIRES].

[7] A. Masiero, M. Piai, A. Romanino and L. Silvestrini, Signals of supersymmetric flavor models in B physics, Phys. Rev. D 64 (2001) 075005 [hep-ph/0104101] [SPIRES].

[8] T. Moroi, CP violation in $B_{d} \rightarrow \varphi K_{S}$ in SUSY GUT with right-handed neutrinos, Phys. Lett. B 493 (2000) 366 [hep-ph/0007328] [SPIRES].

[9] D. Chang, A. Masiero and H. Murayama, Neutrino mixing and large CP-violation in B physics, Phys. Rev. D 67 (2003) 075013 [hep-ph/0205111] [SPIRES].

[10] R. Harnik, D.T. Larson, H. Murayama and A. Pierce, Atmospheric neutrinos can make beauty strange, Phys. Rev. D 69 (2004) 094024 [hep-ph/0212180] [SPIRES].

[11] T. Hurth and W. Porod, Non-diagonal flavour observables in B and collider physics, Eur. Phys. J. C 33 (2004) s764 [hep-ph/0311075] [SPIRES].

[12] S. Dittmaier, G. Hiller, T. Plehn and M. Spannowsky, Charged-Higgs Collider Signals with or without Flavor, Phys. Rev. D 77 (2008) 115001 [arXiv:0708.0940] [SPIRES].

[13] Y. Grossman, Y. Nir, J. Thaler, T. Volansky and J. Zupan, Probing Minimal Flavor Violation at the LHC, Phys. Rev. D 76 (2007) 096006 [arXiv:0706.1845] [SPIRES].

[14] J.L. Feng, C.G. Lester, Y. Nir and Y. Shadmi, The Standard Model and Supersymmetric Flavor Puzzles at the Large Hadron Collider, Phys. Rev. D 77 (2008) 076002 [arXiv:0712.0674] [SPIRES].

[15] A. Crivellin and U. Nierste, Supersymmetric renormalisation of the CKM matrix and new constraints on the squark mass matrices, Phys. Rev. D 79 (2009) 035018 [arXiv:0810.1613] [SPIRES].

[16] G. Bozzi, B. Fuks and M. Klasen, Non-diagonal and mixed squark production at hadron colliders, Phys. Rev. D 72 (2005) 035016 [hep-ph/0507073] [SPIRES].

[17] G. Bozzi, B. Fuks, B. Herrmann and M. Klasen, Squark and gaugino hadroproduction and decays in non- minimal flavour violating supersymmetry, Nucl. Phys. B 787 (2007) 1 [arXiv: 0704.1826] [SPIRES].

[18] B. Fuks, B. Herrmann and M. Klasen, Flavour Violation in Gauge-Mediated Supersymmetry Breaking Models: experimental Constraints and Phenomenology at the $\mathrm{LHC}$, Nucl. Phys. B 810 (2009) 266 [arXiv:0808.1104] [SPIRES].

[19] B. Allanach et al., SUSY Les Houches Accord 2, Comp. Phys. Commun. 180 (2009) 8 [arXiv:0801.0045] [SPIRES].

[20] T. Besmer, C. Greub and T. Hurth, Bounds on supersymmetric flavour violating parameters from $B \rightarrow X_{s} \gamma$, Nucl. Phys. B 609 (2001) 359 [hep-ph/0105292] [SPIRES]. 
[21] M. Ciuchini, E. Franco, A. Masiero and L. Silvestrini, $b \rightarrow s$ transitions: a new frontier for indirect SUSY searches, Phys. Rev. D 67 (2003) 075016 [Erratum ibid. D 68 (2003) 079901] [hep-ph/0212397] [SPIRES].

[22] M. Ciuchini et al., Soft SUSY breaking grand unification: leptons versus quarks on the flavor playground, Nucl. Phys. B $\mathbf{7 8 3}$ (2007) 112 [hep-ph/0702144] [SPIRES].

[23] M. Ciuchini and L. Silvestrini, Upper bounds on SUSY contributions to $b \rightarrow s$ transitions from $B_{s}-\bar{B}_{s}$ mixing, Phys. Rev. Lett. 97 (2006) 021803 [hep-ph/0603114] [SPIRES].

[24] S. Heinemeyer, W. Hollik, F. Merz and S. Penaranda, Electroweak precision observables in the MSSM with non- minimal flavor violation, Eur. Phys. J. C 37 (2004) 481 [hep-ph/0403228] [SPIRES].

[25] J.A. Casas and S. Dimopoulos, Stability bounds on flavor-violating trilinear soft terms in the MSSM, Phys. Lett. B 387 (1996) 107 [hep-ph/9606237] [SPIRES].

[26] J.A. Aguilar-Saavedra et al., Supersymmetry parameter analysis: sPA convention and project, Eur. Phys. J. C 46 (2006) 43 [hep-ph/0511344] [SPIRES].

[27] A. De Roeck et al., Supersymmetric benchmarks with non-universal scalar masses or gravitino dark matter, Eur. Phys. J. C 49 (2007) 1041 [hep-ph/0508198] [SPIRES].

[28] WMAP collaboration, D.N. Spergel et al., Wilkinson Microwave Anisotropy Probe (WMAP) three year results: implications for cosmology, Astrophys. J. Suppl. 170 (2007) 377 [astro-ph/0603449] [SPIRES].

[29] G. Degrassi, S. Heinemeyer, W. Hollik, P. Slavich and G. Weiglein, Towards high-precision predictions for the MSSM Higgs sector, Eur. Phys. J. C 28 (2003) 133 [hep-ph/0212020] [SPIRES].

[30] S. Heinemeyer, W. Hollik and G. Weiglein, Electroweak precision observables in the minimal supersymmetric standard model, Phys. Rept. 425 (2006) 265 [hep-ph/0412214] [SPIRES].

[31] B.C. Allanach, A. Djouadi, J.L. Kneur, W. Porod and P. Slavich, Precise determination of the neutral Higgs boson masses in the MSSM, JHEP 09 (2004) 044 [hep-ph/0406166] [SPIRES].

[32] S. Heinemeyer, W. Hollik, D. Stöckinger, A.M. Weber and G. Weiglein, Precise prediction for $M_{W}$ in the MSSM, JHEP 08 (2006) 052 [hep-ph/0604147] [SPIRES].

[33] Particle Data Group collaboration, C. Amsler et al., Review of particle physics, Phys. Lett. B 667 (2008) 1 [SPIRES].

[34] M. Misiak et al., The first estimate of $\mathcal{B}\left(\bar{B} \rightarrow X_{s} \gamma\right)$ at $O\left(\alpha_{s}^{2}\right)$, Phys. Rev. Lett. 98 (2007) 022002 [hep-ph/0609232] [SPIRES].

[35] E. Lunghi, W. Porod and O. Vives, Analysis of enhanced $\tan \beta$ corrections in MFV GUT scenarios, Phys. Rev. D 74 (2006) 075003 [hep-ph/0605177] [SPIRES].

[36] P.L. Cho, M. Misiak and D. Wyler, $K_{L} \rightarrow \pi^{0} e^{+} e^{-}$and $B \rightarrow X_{s} \ell^{+} \ell^{-}$Decay in the MSSM, Phys. Rev. D 54 (1996) 3329 [hep-ph/9601360] [SPIRES].

[37] T. Hurth, E. Lunghi and W. Porod, Untagged $\bar{B} \rightarrow X_{s+d} \gamma C P$ asymmetry as a probe for new physics, Nucl. Phys. B 704 (2005) 56 [hep-ph/0312260] [SPIRES].

[38] A.J. Buras, P.H. Chankowski, J. Rosiek and L. Slawianowska, $\Delta M_{d, s}, B_{d, s}^{0} \rightarrow \mu^{+} \mu^{-}$and $B \rightarrow X_{s} \gamma$ in supersymmetry at large $\tan \beta$, Nucl. Phys. B 659 (2003) 3 [hep-ph/0210145] [SPIRES]. 
[39] T. Huber, T. Hurth and E. Lunghi, Logarithmically Enhanced Corrections to the Decay Rate and Forward Backward Asymmetry in $\bar{B} \rightarrow X_{s} \ell^{+} \ell^{-}$, Nucl. Phys. B 802 (2008) 40 [arXiv: 0712.3009] [SPIRES].

[40] W. Porod, SPheno, a program for calculating supersymmetric spectra, SUSY particle decays and SUSY particle production at $e^{+} e^{-}$colliders, Comput. Phys. Commun. 153 (2003) 275 [hep-ph/0301101] [SPIRES].

[41] D.M. Pierce, J.A. Bagger, K.T. Matchev and R.-j. Zhang, Precision corrections in the minimal supersymmetric standard model, Nucl. Phys. B 491 (1997) 3 [hep-ph/9606211] [SPIRES].

[42] W. Porod, Including flavour violation and R-parity violation in the numerical computation of the spectrum of supersymmetric models: SPheno3.0, in preparation.

[43] E. Lunghi and J. Matias, Huge right-handed current effects in $B \rightarrow K *(K \pi) \ell^{+} \ell^{-}$in supersymmetry, JHEP 04 (2007) 058 [hep-ph/0612166] [SPIRES].

[44] C. Bobeth, A.J. Buras, F. Krüger and J. Urban, $Q C D$ corrections to $\bar{B} \rightarrow X_{d, s} \nu \bar{\nu}$, $\bar{B}_{d, s} \rightarrow \ell^{+} \ell^{-}, K \rightarrow \pi \nu \bar{\nu}$ and $K_{L} \rightarrow \mu^{+} \mu^{-}$in the MSSM, Nucl. Phys. B 630 (2002) 87 [hep-ph/0112305] [SPIRES].

[45] S. Baek, T. Goto, Y. Okada and K.-i. Okumura, Muon anomalous magnetic moment, lepton flavor violation and flavor changing neutral current processes in SUSY GUT with right-handed neutrino, Phys. Rev. D 64 (2001) 095001 [hep-ph/0104146] [SPIRES].

[46] T. Huber, E. Lunghi, M. Misiak and D. Wyler, Electromagnetic logarithms in $\bar{B} \rightarrow X_{s} \ell^{+} \ell^{-}$, Nucl. Phys. B $\mathbf{7 4 0}$ (2006) 105 [hep-ph/0512066] [SPIRES].

[47] J. Cao, G. Eilam, K.-i. Hikasa and J.M. Yang, Experimental constraints on stop-scharm flavor mixing and implications in top-quark FCNC processes, Phys. Rev. D 74 (2006) 031701 [hep-ph/0604163] [SPIRES].

[48] J.J. Cao et al., Supersymmetry-induced flavor-changing neutral-current top-quark processes at the CERN Large Hadron Collider, Phys. Rev. D 75 (2007) 075021 [hep-ph/0702264] [SPIRES].

[49] W. Porod and T. Wohrmann, Higher order top squark decays, Phys. Rev. D 55 (1997) 2907 [Erratum ibid. D 67 (2003) 059902] [hep-ph/9608472] [SPIRES].

[50] W. Porod, More on higher order decays of the lighter top squark, Phys. Rev. D 59 (1999) 095009 [hep-ph/9812230] [SPIRES].

[51] A. Djouadi and Y. Mambrini, Three-body decays of top and bottom squarks, Phys. Rev. D 63 (2001) 115005 [hep-ph/0011364] [SPIRES].

[52] C. Boehm, A. Djouadi and Y. Mambrini, Decays of the lightest top squark, Phys. Rev. D 61 (2000) 095006 [hep-ph/9907428] [SPIRES].

[53] ATLAS ANd CMS collaboration, J.G. Branson et al., High transverse momentum physics at the Large Hadron Collider: the ATLAS and CMS Collaborations, Eur. Phys. J. direct C 4 (2002) N1 [hep-ph/0110021] [SPIRES].

[54] A. Bartl et al., Impact of squark generation mixing on the search for gluinos at LHC, arXiv: 0905.0132 [SPIRES].

[55] J. Hisano, K. Kawagoe and M.M. Nojiri, A detailed study of the gluino decay into the third generation squarks at the CERN LHC, Phys. Rev. D 68 (2003) 035007 [hep-ph/0304214] [SPIRES]. 
[56] A. Bartl et al., Search of stop, sbottom, $\tau$-sneutrino and stau at an $e^{+} e^{-}$linear collider with $\sqrt{s}=0.5-2 \mathrm{TeV}$, Z. Phys. C 76 (1997) 549 [hep-ph/9701336] [SPIRES].

[57] G.A. Moortgat-Pick et al., Polarized positrons and electrons at the linear collider, Phys. Rept. 460 (2008) 131 [hep-ph/0507011] [SPIRES].

[58] LHC/LC Study Group collaboration, G. Weiglein et al., Physics interplay of the LHC and the ILC, Phys. Rept. 426 (2006) 47 [hep-ph/0410364] [SPIRES].

[59] CLIC Physics Working Group collaboration, E. Accomando et al., Physics at the CLIC multi-TeV linear collider, hep-ph/0412251 [SPIRES]. 